\title{
Pegylated Granulocyte Colony Stimulating Factor MAXY-G34
}

National Cancer Institute

\section{Source}

National Cancer Institute. Pegylated Granulocyte Colony Stimulating Factor MAXY-G34.

NCI Thesaurus. Code C71718.

\begin{abstract}
A long-acting, pegylated recombinant variant of human granulocyte colony-stimulating factor (G-CSF) with immunomodulating activity. Pegylated granulocyte colony stimulating factor MAXY-G34 contains multiple non-naturally occurring lysines that have been introduced into alpha helixes of wild type human G-CSF as pegylation sites; other naturally occurring lysine residues have been removed to prevent pegylation and the variant G-CSF is pegylated with methoxypolyethylene glycol succinimidyl propionate (MPEG SPA) at three amino acid residues. Similar to endogenous G-CSF, this agent binds to and activates specific cell surface receptors, stimulating neutrophil progenitor proliferation and differentiation and selected neutrophil functions. A difference in the degree of pegylation may account for the extended half-life of this agent compared to pegfilgrastim which is pegylated at only one amino acid residue.
\end{abstract}

